\title{
A Discrete-Time Equivalent System Approach to the Periodic Response of Nonlinear Autonomous Circuits
}

\author{
P. Palà Schönwälder and J. M. Miró Sans \\ UPC-Department of Signal Theory and Communications \\ PO Box 30.002 \\ 08080 Barcelona - Spain \\ (+34) 34017072 \\ pere@tsc.upc.es
}

\begin{abstract}
The problem of computing the steady state response of nonlinear autonomous circuits is solved making use of a discrete-time equivalent system approach. With the application of an $s$-plane to $z$-plane mapping, the circuit equations are discretized and written in vector form. Using this technique, it is not necessary to repeatedly compute transforms between the time and the frequency domain. An efficient scheme to build the jacobian matrix with exact partial derivatives with respect to the oscillation period and with respect to the samples of the unknown variables is described. Application examples on two widely studied circuits are provided to validate the proposed technique.
\end{abstract}

\section{INTRODUCTION}

Several techniques have been developed to solve the problem of determining the steady-state response of nonlinear autonomous circuits. An important class of timedomain methods for the steady-state analysis of forced nonlinear circuits are the so-called shooting methods which try to find the initial conditions that make zero the transient response. Although this technique has been optimized successively, its application is expensive in terms of computing time. Additional difficulties arise in the case of autonomous circuits where there is no a-priori knowledge of the oscillation period.

Frequency-domain methods divide the circuit into a linear and a nonlinear part, taking advantage of the high efficiency than may be achieved solving the linear part. However, as the nonlinearities are best evaluated in the time domain, it is necessary to take successive transformations between both domains, consuming a substantial amount of time while limiting accuracy. Extension to autonomous circuits requires the computation of sensitivities with respect to the oscillation period in order to be able to imbed this added unknown into an iterative solving scheme. It should be pointed out that when the circuit variables have a broad spectrum -i.e., relaxation oscillators- the number of harmonics required to satisfactorily approximate the waveforms may be very high, requiring excessive computer time.

In this paper we describe an efficient technique for solving the steady-state response of nonlinear autonomous circuits based on the formulation of the circuit equations in the time domain, making it unnecessary to take any further transformation. After discretizing the equations with the use of an $s$-plane to $z$-plane mapping and the application of the inverse- $z$ transform, the problem is written in vector form, making it possible to take advantage of parallel processing techniques. An extremely efficient method for the computation of the jacobian matrix, with exact partial derivatives with respect to the samples of the circuit variables and the oscillation period, has been implemented.

\section{EQUATION FORMULATION}

Consider a nonlinear autonomous circuit where all bias sources and all nonlinear elements have been extracted. Every nonlinear element may be modeled by the parallel combination of a linear element and a nonlinear controlled source [1]. Figure 1 depicts an idealized example where only one bias source $u_{b}$ and one nonlinear element described by the nonlinear function $i=F(x)$ are included for the sake of simplicity. The extension to an arbitrary number of bias sources and nonlinear elements is straightforward.

By application of superposition, the control variable of the nonlinearity $x$ and the desired output variable $y$ may be expressed as 


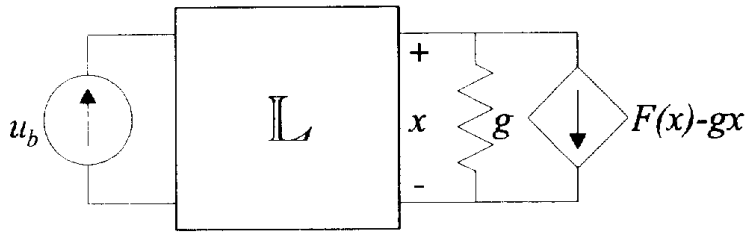

Figure 1

$$
\begin{aligned}
& x=A\left\{u_{b}\right\}+B\{F(x)\} \\
& y=C\left\{u_{b}\right\}+D\{F(x)\}
\end{aligned}
$$

where $A, B, C$ and $D$ represent linear operators. In the case of dynamic circuits, the terms $A\left\{u_{b}\right\}, B\{F(x)\}$ correspond to convolutions in the time domain. The evaluation of $v=A\left\{u_{b}\right\}$ is simple, since it is a linear problem. The resulting problem is then to solve $x=v+B\{F(x)\}$ for the variable $x$, assumed to be periodic with unknown period $T$. This equation may be expressed in the time domain as

$$
x(t)=v(t)+\int_{-\infty}^{t} b(t-\tau) f(x(\tau)) d \tau
$$

where $b(t)$ is the impulse response of the linear circuit $\mathbb{L}$ to the excitation of the nonlinear source.

An efficient way to solve this integral equation consists in discretizing the analog convolution. To obtain the equivalent discrete intrinsic impulse response $b(n)$, Frey and Norman [1] propose to first apply an $s$-plane to $z$-plane mapping to the Laplace transform of $b(t)$. The resulting function of $z$ is then inverse- $z$ transformed to the sequence domain. In this process, it is important that the number of samples per period $N$ satisfies the Nyquist criterion to prevent aliasing. The application of this strategy allows to express (1) as

$$
x(n)=v(n)+\sum_{k=-\infty}^{n} b(n-k) f(x(k))
$$

Since the variables are periodic with period $T$, it is possible to rewrite (2) in vector form as

$$
\mathbf{x}=\mathbf{v}+\mathbf{B} \mathbf{f}(\mathbf{x})
$$

where $x=\left[x_{1}, x_{2}, \ldots, x_{N}\right]^{t}, v=\left[v_{1}, v_{2}, \ldots, v_{N}\right]^{t}, f(x)=\left[f\left(x_{1}\right)\right.$, $\left.f\left(x_{2}\right), \ldots, f\left(x_{N}\right)\right]^{t}$ and $B$ is a circulant matrix that operates on a vector of samples $\mathbf{x}$ in the equivalent form as the analog operator $B(s)$ operates on the signal $x(t)$. The matrix $\mathbf{B}$ may be computed as follows.
In general, $B(s)=n(s) / d(s)$. Then, after applying the map $s=T\left(z^{-1}\right)$, a rational transfer function $B(z)=q\left(z^{l}\right) / p\left(z^{1}\right)$ is obtained. The effect of $B(z)$ on a vector of input samples may be described by the difference equation

$$
\begin{aligned}
& p_{0} y(n)+p_{1} y(n-1)+\cdots+p_{k} y(n-k)= \\
& q_{0} x(n)+q_{1} x(n-1)+\cdots+q_{r} x(n-r)
\end{aligned}
$$

which, in the case of periodic sequences, may be expressed in vector form

$$
p_{0} \mathbf{y}+p_{1} \mathbf{S y}+\cdots+p_{k} \mathbf{S}^{k} \mathbf{y}=q_{0} \mathbf{x}+q_{1} \mathbf{S} \mathbf{x}+\cdots+q_{r} \mathbf{S}^{r} \mathbf{x}
$$

where $\mathbf{S}$ is the matrix circular shift operator, $S=\operatorname{circ}\left[\begin{array}{lll}0 & 1 & 0\end{array}\right.$ $0]$. Finally, $\mathbf{P y}=\mathbf{Q x}$, and the output sequence may be obtained as $\mathbf{y}=\mathbf{B x}$, with $\mathbf{B}=\mathbf{P}^{-1} \mathbf{Q}$.

To obtain the steady-state response, it is necessary to find the solution of the nonlinear set of equations (3), where the unknowns are the $N$ samples of the control variable $x$ and the period of oscillation $T$. The fact that the oscillation period $T$ is an unknown is an added difficulty with respect to the analysis of forced circuits where, in general, the period of the variables is fixed by the external signal sources. Having $N+1$ unknowns and only $N$ restrictions indicates the presence of an infinite number of solutions. In particular, note that if a vector $x_{p}$ is a solution of (3), $S^{q} x_{p}$ is also a solution for any integer $q$. The existence of a continuum of solutions makes it possible to fix the value of one of the samples of $x$ [2]. In the following, and without loss of generality, the value of the first sample $x_{1}$ will be assumed fixed.

In the case of forced circuits, it is possible to implement methods for solving (3) based on relaxation strategies, taking advantage of the resulting parallelism. However, this approach is not so appealing in the autonomous case because of the difficulty of adequately embedding the period $T$ in the relaxation scheme. The efficient method for computing the partial derivatives with respect to the samples and with respect to the period to be described next makes it particularly interesting to make use of higher order solution methods that make use of the jacobian matrix.

\section{COMPUTATION OF PARTLAL DERIVATIVES}

To simplify the computation of the partial derivatives with respect to the oscillation period $T$, it is convenient to reformulate the initial equation (3). An equivalent formulation is

$$
\mathbf{P}(T) \mathbf{x}-\mathbf{P}(T) \mathbf{v}-\mathbf{Q}(T) \mathbf{f}(\mathbf{x})=\mathbf{0}
$$

which may be expressed as 


$$
\mathbf{F}\left(T, x_{2}, \cdots, x_{N}\right)=\left\{\begin{array}{c}
f_{1}\left(T, x_{2}, \cdots, x_{N}\right)=0 \\
\vdots \\
f_{N}\left(T, x_{2}, \cdots, x_{N}\right)=0
\end{array}\right.
$$

The dependence of (4) with the period appears implicitly when the $s$-plane to $z$-plane mapping is performed. In every mapping $s=T_{0}(z) / \Delta[2]$, the dependence with the oscillation period $T$ appears in the term $\Delta=T / N$. In our work, the second order Gear map $s=\left(3-4 z^{-1}+z^{-2}\right) /(2 \Delta)$ has been used. To compute the partial derivative of the $k$-th equation with respect to the period, some previous steps are necessary.

Let $P_{n}(s)$ be a generic polynomial in the variable $s$

$$
P_{n}(s)=p_{n} s^{n}+p_{n-1} s^{n-1}+\cdots+p_{1} s+p_{0}
$$

After applying the mapping $s=T_{0}(z) / \Delta$, the following expression is obtained.

$$
\begin{aligned}
P_{n}(z) & =\frac{p_{n}}{\Delta^{n}} T_{0}(z)^{n}+\frac{p_{n-1}}{\Delta^{n-1}} T_{0}(z)^{n-1}+\cdots+\frac{p_{1}}{\Delta} T_{0}(z)+p_{0}= \\
& =\hat{p}_{0}+\hat{p}_{1} z^{-1}+\cdots+\hat{p}_{m} z^{-m}
\end{aligned}
$$

where, for the Gear-2 map, $m=2 n$. The associated matrix is then obtained as

$$
\mathbf{P}=\sum_{k=0}^{m} \hat{p}_{k} \mathbf{S}^{k}
$$

Now,

$$
\begin{aligned}
\frac{\mathscr{P}_{n}(z)}{\partial T} & =\frac{-1}{N}\left[\frac{n p_{n}}{\Delta^{n+1}} T_{0}(z)^{n}+\frac{(n-1) p_{n-1}}{\Delta^{n}} T_{0}(z)^{n-1}+\cdots+\frac{p_{1}}{\Delta^{2}} T_{0}(z)\right]= \\
& =\hat{p}_{0}^{\prime}+\hat{p}_{1}^{\prime} z^{-1}+\cdots+\hat{p}_{m}^{\prime} z^{-m}
\end{aligned}
$$

and the associated matrix may be computed as

$$
\mathbf{P}=\sum_{k=0}^{m} \hat{p}_{k}^{\prime} \mathbf{S}^{k}
$$

Using this notation, the partial derivative of (5) with respect to the period of oscillation is obtained as

$$
\frac{\partial f_{i}}{\partial T}=\sum_{k=1}^{N} p_{j k}^{\prime} x_{k}-\sum_{k=1}^{N} p_{i k}^{\prime} v_{k}-\sum_{k=1}^{N} q_{i k}^{\prime} f\left(x_{k}\right)
$$

Finally, the jacobian matrix $\mathbf{J}$ is obtained as

$$
\mathbf{J}_{i, 1}=\frac{\partial f_{i}}{\partial T}
$$

$$
\mathbf{J}_{1: N, 2: N}=\mathbf{P}_{1: N, 2: N}+\mathbf{Q}_{1: N, 2: N} \operatorname{diag}\left(f^{\prime}\left(x_{2}\right), \cdots, f^{\prime}\left(x_{N}\right)\right)
$$

where $f^{\prime}\left(x_{k}\right)=d f(x) /\left.d x\right|_{x=x k}$

For implementation purposes, it is possible to save a significant amount of computer memory taking advantage of the fact that the circulants $\mathbf{P}$ and $\mathbf{Q}$ only have $N$ different entries. It should be also pointed out that the special structure of $\mathbf{J}$ allows for efficient LU decomposition coding. Also, since in the autonomous case there is no external signal input, the vector $\mathbf{v}$ is constant as it is the response due to the bias sources. The result of applying $T_{0}{ }^{k}\left(z^{-1}\right)$ on a vector of constant samples is a zero vector, except for $k=0$.

The inexpensive availability of the exact jacobian matrix allows to implement efficient algorithms for solving the nonlinear system of equations (3). It is well-known that Newton's method converges quadratically when the starting point is sufficiently close to the solution but may even not converge on certain situations. To overcome this difficulty, globally convergent modifications of Newton's method [3] have been implemented.

\section{APPLICATION EXAMPLES}

To validate the described technique, its application to two kinds of widely studied classic oscillator circuits is presented.

\section{Van der Pol oscillator}

The van der Pol equation

$$
\ddot{x}+\mu\left(x^{2}-1\right) \dot{x}+x=0
$$

may be modeled as depicted in figure 2 .

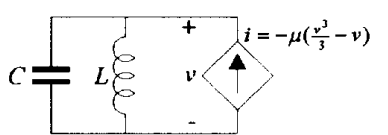

Fig. 2
Depending on the value of parameter $\mu$, the circuit behavior for fixed values of $L$ and $C(L=1, C=1)$ ranges from a nearly sinusoidal oscillator for small values of $\mu(\mu-0.1)$ to a relaxation oscillator ( $\mu \sim$ 4.10 ), with an important number of significant harmonics. Applying harmonic balance techniques requires to consider an important number of harmonics - with the corresponding computational cost - in order to achieve sufficient accuracy.

The waveforms resulting for different values of $\mu$ along with the corresponding oscillation period have been obtained with the described technique. The resulting waveforms and periods have been compared with the results obtained with 


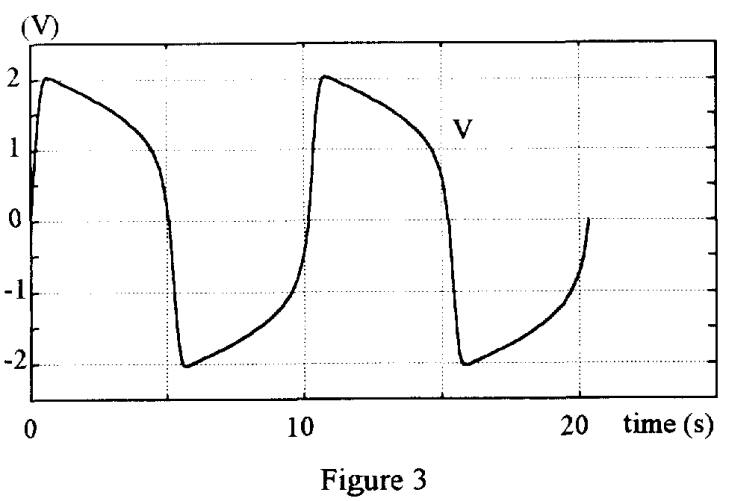

the use of time-domain integration methods. The obtained period has also been compared with the values given in reference [4]. Good agreement has been observed in both cases. The results obtained in [5] using a harmonic balance approach show significantly more distortion with a comparable computational cost. For illustration purposes, the obtained waveform for $\mu=5$ is depicted in figure 3 .

\section{Colpitts oscillator}

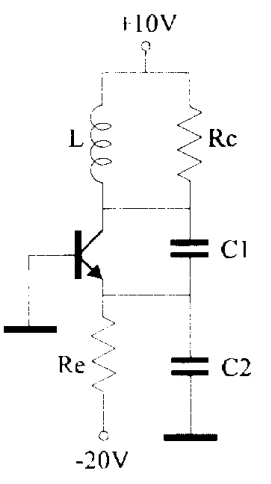

Fig. 4
The Colpitts oscillator in figure 4 has been analyzed using the described technique, with normalized element values $R c=10, L=0.1, C l=2$, $C 2=0.8$ and $R e=20$. For the bipolar transistor the EbersMoll model with parameters $I c s=462 \mathrm{E}-12$, Ies $=7 \mathrm{E}-12$, $\alpha_{\mathrm{F}}=0.99, \quad \alpha_{\mathrm{R}}=0.015 \quad$ and $\mathrm{V}_{\mathrm{T}}=0.025$ has been used. This circuit presents a relatively high $Q$ factor. For this reason classical time domain integration methods are less

efficient. However, the waveforms obtained with these methods agree with the results obtained from the described technique. In this example $N=30$ samples per period have been used. The implemented solution algorithm started with $1 \mathrm{~V}$ amplitude sinusoidal waveforms with $T=1 \mathrm{~s}$ for both unknowns $V_{c b}$ and $V_{c b}$ with a dc component of $10 \mathrm{~V}$ for $V_{c b}$ obtained from circuit inspection. The final oscillation period was $T=1.52 \mathrm{~s}$ and the resulting waveforms are shown in figure 5 .

\section{CONCLUSIONS}

In this paper, an efficient technique for the analysis of nonlinear autonomous circuits has been described. The

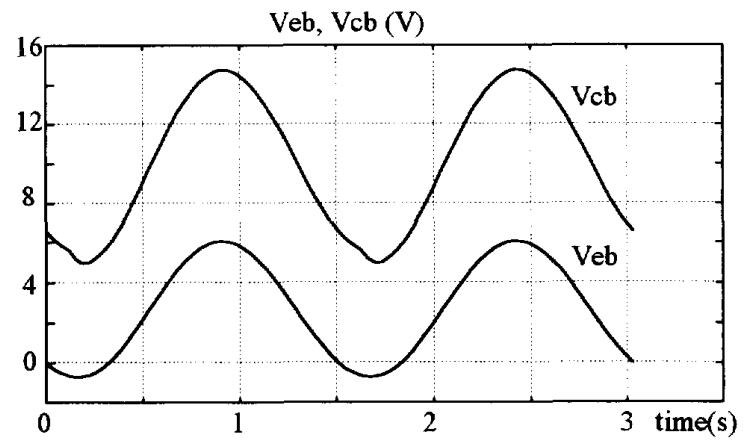

Figure 4

method is based on the formulation and subsequent discretization of the circuit equations in the time domain, so that it is not necessary to perform any additional transform between the time and frequency domains. The discretized equations are then written in vector form, allowing to take advantage of parallel processing. In the resulting formulation, the unknowns to be solved for simultaneously are the samples of the control variables of the nonlinear elements and the oscillation period. An efficient method for constructing the jacobian matrix with exact computation of the sensitivities with respect to the samples and the period is described.

\section{REFERENCES}

[1] D. Frey and O. Norman, "An integral equation approach to the periodic steady-state problem in nonlinear circuits", IEEE Trans. Circuits and Systems, vol. 39, pp. 744-755, September 1992.

[2] L. Chua, P. Lin, Computer-Aided Analysis of Electronic Circuits, Englewood Cliffs, NJ: Prentice Hall, 1975.

[3] J. E. Dennis and R. B. Schnabel, Numerical Methods for Unconstrained Optimization and Nonlinear Equations, Englewood Cliffs, NJ, Prentice Hall, 1983.

[4] D. Hente and R. H. Jansen, "Frequency domain continuation method for the analysis and stability investigation of nonlinear microwave circuits", Proc. $I E E$, vol. 133, Pt. H. pp. 351-362, October 1986.

[5] E. Martin, Analysis of Nonlinear Autonomous Circuits with Harmonic Balance Techniques, $\mathrm{Ph}$. D. Thesis, Madrid, September 1990. 